\title{
Prevalence of Metabolic Syndrome amongst Bipolar Patients on Lithium and Sodium Valproate Monotherapy
}

\author{
Adam Abba-Aji* \\ Department of Psychiatry, University of Alberta Faculty of Medicine and Dentistry, Edmonton, Alberta, Canada
}

\begin{abstract}
There is a growing body of evidence on metabolic syndrome associated with antipsychotic medications. However, very limited data exist on metabolic syndrome and commonly prescribed mood stabilizers such as Lithium, Valproic acid and Carbamazepine.

Method: This paper is a cross sectional survey of metabolic syndrome in a group of patients diagnosed with bipolar affective disorder (BPAD). The study completed a data on 32 patients, 19 females and 13 males. These patients were diagnostically stratified using ICD 10 into BPAD type 1(10) and BPAD type 2 (22). The groups were further divided into two according to their treatment with 19 patients receiving Lithium monotherapy and 13 Valproic acid monotherapy. The International Diabetic Federation (IDF) was used to screen all the patients for metabolic syndrome.
\end{abstract}

Result: The result shows that Lithium has low prevalence of metabolic syndrome (10\%) while Valproic acid possessed significant risk with a prevalence rate of $30 \%$.

Conclusion: It is therefore important to extend metabolic monitoring beyond antipsychotics to cover mood stabilizing agents especially Valproic acid.

Limitation: Small sample size. However, it is very difficult to find bipolar patients on a monotherapy of mood stabilizer.

Keywords: Lithium; Valproic acid; Bipolar disorder; Metabolic syndrome

\section{Introduction}

The world of psychiatry had witnessed phenomenal development towards diagnosis and treatment of mental disorders. However, the wind of change came with the inevitable side effects of medications. Decades ago the extrapyramidal side effects of antipsychotic medications possess a significant challenge for both the clinicians and indeed the patients. Such a challenge had resulted in poor medication compliance, poor outcome, secondary negative symptoms, cognitive impairment, Parkinsonism, and depression [1]. Several studies have demonstrated that mental illness, including schizophrenia, bipolar disorder and depression are associated with excess medical mortality [2]. This increased mortality had been observed consistently across various populations, with similar findings reported in North America and Western Europe. In schizophrenia, it was reported that $80 \%$ of patients die of natural causes compared with $97 \%$ of the general population [3]. Osby et al. reported that cardiovascular disease (CVD) was the most common cause of mortality among patients with schizophrenia, accounting for $34 \%$ of deaths among male patients and $31 \%$ in female patients. The increased mortality from vascular disease among patients with schizophrenia, bipolar disorder and depression is highly correlated with established risk factors such as increased weight, insulin resistance, an elevated glucose and lipids levels in patients with mental illness.

Metabolic syndrome has been used to describe a set of commonly co-occurring conditions that include obesity (particularly abdominal), insulin resistance, impaired glucose tolerance, disturbances in uric acid metabolism, hypertension, and prothrombotic and pro-inflammatory states, which can increase the risk of CVD [4]. Its incidence and prevalence have risen dramatically, especially in the western countries, as a result of the increasing prevalence of obesity [5]. As in schizophrenia, there is great concern about the prevalence of metabolic syndrome in patients with bipolar disorder, although research in this area is scarce [6]. Study by Fagiolini et al. [7] of 171 subjects revealed a prevalence of metabolic syndrome in bipolar disorders as high as $30 \%$ compared to the general population.

In the United States, the risk of type 2 diabetes is growing to epidemic magnitude. However, many physicians are unaware about the association between bipolar disorder (as supposed to schizophrenia) and increased risk of diabetes [8]. McEvoy et al. reported that metabolic syndrome is common occurrence in patients with Severe Mental Illnesses (SMI) and the prevalence is in the range of $30-60 \%$ for schizophrenic and bipolar disorders. The high prevalence of sedentary lifestyle, smoking and poor diet contributes further to physical morbidity among this group of patients [2]. Osby further suggested that Schizophrenics, in particular, die at least 10 years earlier than age matched contemporaries and have an increased relative risk of premature death by two to four folds.

A number of experts group have developed clinical criteria for the metabolic syndrome. The most widely accepted of these were produced by the International Diabetes Federation (IDF), European Group for the Study of Insulin Resistance (EGIR), and the National Cholesterol

*Corresponding author: Adam Abba-Aji, Department of Psychiatry, University of Alberta Faculty of Medicine and Dentistry, Edmonton, Alberta, Canada, Tel: +7803425091; E-mail: abbaaji@ualberta.ca

Received: July 16, 2017; Accepted: August 04, 2017; Published: August 11, 2017

Citation: Abba-Aji A (2017) Prevalence of Metabolic Syndrome amongst Bipolar Patients on Lithium and Sodium Valproate Monotherapy. J Psychiatry 20: 415 doi:10.4172/2378-5756.1000415

Copyright: (c) 2017 Abba-Aji A. This is an open-access article distributed under the terms of the Creative Commons Attribution License, which permits unrestricted use, distribution, and reproduction in any medium, provided the original author and source are credited 
Education Program - Third Adult Treatment Panel (NCEP ATP III) and the World Health Organisation (WHO) clinical criteria for diagnosing the metabolic syndrome [9]. The diagnostic framework adopted by WHO specified the risk factors that can predict either the development of type 2 diabetes mellitus or cardiovascular disease (CVD). Those components combined into a unique clinical entity, and the patients should be considered to have the metabolic syndrome. International Diabetes Federation (IDF) has reflects a consensus of 21 different countries. The IDF's definition of the metabolic syndrome highlights additional metabolic criteria which should be measured (IDF 2005) (Table 1). Illustrates the components that make up the diagnostic criteria for the IDF version of the metabolic syndrome.

It is unknown why some patients will develop metabolic syndrome while others will not [10]. Bobes et al. reported that the risk of coronary heart disease and stroke was found to be tripled among subjects with metabolic syndrome as compared with those who did not have metabolic syndrome $(\mathrm{p}<0.001)$. There is a statistically significant association between atypical antipsychotics and metabolic syndrome. However, there are no such association between mood stabilizers and metabolic syndrome [6]. This study evaluates the prevalence of metabolic syndrome in 32 patients with bipolar disorder that are either on Li or VPA monotherapy.

\section{Methodology}

The study was a cross-sectional survey and was approved by the local ethics committee. All patients must sign the informed consent forms. Fifty-two patients attending the community mental health services (CMHS) were consecutively enrolled from January 2008 to February 2009. Patients included in this study has a diagnosed of bipolar affective according to ICD-10 criteria and were required to be only on either lithium (Li) or Valproic acid (VPA) monotherapy. All subjects were aged $18-65$ years old.

Patients with the following conditions were excluded: (1) ICD-10 diagnosis of substance abuse within past three months, (2) Organic mental diseases, mental retardation or dementia, (3) Pre-existing physical illness other than hypertension or dyslipidemias prior to commencement of Li or VPA, (4) Pre-existing obesity prior to commencement of Li or VPA, (5) Other psychotropic medications other than Li or VPA and (6) Use of antipsychotic medications for more than one month in the last three months.

Patient's medical record, information from relatives and General Practitioners records were used to validate subjective recalls. Data was collected on all patients. The data was divided into three segments, the demographics, biomedical measurements and life styles (amount of cigarette per day and number of hours of exercise per week). The demographics included gender, age, diagnosis, age of onset and duration of illness, family history of diabetes or CVD. Biochemical and anthropometric measurements complied with the measurement of the metabolic syndrome International Diabetes Federation (IDF) 2005 criteria. The body weight (to the nearest $0.1 \mathrm{~kg}$ ) was measured using the metric measuring scale. Pre-Li/NaVP weight was determined and validated from patient's medical record, information from relatives and General Practitioners records. The waist circumference was measured at the level midway between the lateral lower rib margin and the superior anterior iliac crest. Metabolic syndrome was defined according to the IDF 2005 criteria (Table 1).

Blood was taken for testing from all patients. Whole venous blood samples were taken into tubes containing ethylenediamine tetra-acetic acid (EDTA) at approximately 08:30 h following overnight fasting. Plasma glucose concentration was assayed by an automated glucose oxidase method. Total high-density lipoprotein (HDL) and low density lipoprotein (LDL) cholesterol and triglycerides were determined by standard clinical biochemistry laboratory assays. Life styles measures included tobacco smoking per week and exercise per week.

\section{Statistical analysis}

Data were analyzed using the Statistical Package for Social Sciences (SPSS 15). Categorical variables were expressed as numbers and percentages, and continuous variables in means \pm SD. The variables were assessed using chi-square test, Student's t-test, and one-way ANOVA. The difference between the groups was considered significant if $\mathrm{p}$-value is 0.05 or less.

\section{Results}

52 patients entered the study. However, 32 patients completed the study and 19 patients were excluded because their subjective information could not be validated. Those who completed the study include 19 females and 13 males with mean age of 36.33 years. Further sub-division of their treatment revealed that $19(59.4 \%)$ patients were on Li monotherapy of which 11 were females and 8 were males. There were $13(40.6 \%)$ patients on VPA monotherapy which include 8 females and 5 males.

There were $10(28 \%)$ patients with bipolar affective disorder (BPAD) type 1 and 8 of them were on Li and 2 on VPA (Table 2). Similarly, there were $22(72 \%)$ diagnosed with BPAD type2 with equal number of patients each on $\mathrm{Li}(\mathrm{n}=11)$ and on VPA $(\mathrm{n}=11)$ monotherapy. The patients with mean duration of illness were 12.44 years and mean age of onset was 23.89 years.

The mean dosage of Li was $900 \mathrm{mg} /$ day and the mean serum level was $0.65 \mathrm{mEq} / \mathrm{l}$. The mean duration of Li was 23.4 months. Similarly, the mean dosage of VPA was $1015 \mathrm{mg} /$ day with mean of 19 months for duration on VPA at mean of $80.7 \mathrm{mEq} / \mathrm{l}$ serum levels.

The prevalence of metabolic syndrome among patients on VPA was found to be $30.8 \%$ ( $\mathrm{p}=0.034$ ), being higher in women $(47.1 \%$ vs. $19.9 \%$, $\mathrm{p}=0.003$ ). The prevalence of metabolic syndrome when stratified by psychiatric diagnostic was $58.1 \%$ for BPAD type 2 and $41.9 \%$ for BPAD type 1 .

After using the multivariate analysis, female gender and VPA more than 6 months remained as factors associated with a diagnosis of metabolic syndrome.

The prevalence of metabolic syndrome among Li monotherapy was $10.5 \%(\mathrm{p}=0.07)$ and no statistically significant difference between the gender and diagnosis.

High Triglycerides occurred in $21 \%$ of the patients with the mean higher in VPA ( $2.5 \mathrm{mmol} / \mathrm{l})$ than Li. High FBG was only found in VPA patients and the mean FBG was remarkably $8.9 \mathrm{mmol} / \mathrm{l}$. The large waist circumference was seen in $25 \%$ of the patients and there is no significant difference between $\mathrm{Li}$ and VPA. The results in Table 3 showed a statistically significant difference of weight gained after commencement of $\mathrm{Li}$ and VPA. About $84 \%(\mathrm{p}<0.002)$ and mean increase of $9.8 \mathrm{~kg}$ in Li and $15 \mathrm{~kg}$ in VPA. In both groups significant weight gain was found to at $>6$ months duration on Li and VPA monotherapy.

Life style measures were based on number of hours of exercise per week and number of cigarettes smoked per day. Overall the mean hours of exercise were 4.6 and 3.9 hours. Interestingly, same average number 


\section{According to the new IDF definition, for a person to be defined as having the metabolic syndrome they must have}

\begin{tabular}{|c|c|}
\hline $\begin{array}{c}\text { Central obesity (defined as waist circumference } \geq 94 \mathrm{~cm} \text { for Europid men } \\
\text { and } \geq 80 \mathrm{~cm} \text { for Europid women, with ethnicity specific values for other } \\
\text { groups) plus any two of the following four factors: }\end{array}$ & $\begin{array}{c}\text { Raised TG level: } \geq 150 \mathrm{mg} / \mathrm{dL}(1.7 \mathrm{mmol} / \mathrm{L}) \text {, or specific treatment for this } \\
\text { lipid abnormality } \\
\text { Reduced HDL cholesterol: }<40 \mathrm{mg} / \mathrm{dL}\left(1.03 \mathrm{mmol} / \mathrm{L}^{*}\right) \text { in males and } \\
<50 \mathrm{mg} / \mathrm{dL}\left(1.29 \mathrm{mmol} / \mathrm{L}^{*}\right) \text { in females, or specific treatment for this lipid } \\
\text { abnormality } \\
\text { Raised blood pressure: systolic } \mathrm{BP} \geq 130 \text { or diastolic } \mathrm{BP} \geq 85 \mathrm{~mm} \mathrm{Hg} \text {, or } \\
\text { treatment of previously diagnosed hypertension } \\
\text { Raised fasting plasma glucose }(\mathrm{FPG}) \geq 100 \mathrm{mg} / \mathrm{dL}(5.6 \mathrm{mmol} / \mathrm{L}) \text {, or previously } \\
\text { diagnosed type } 2 \text { diabetes If above } 5.6 \mathrm{mmol} / \mathrm{L} \text { or } 100 \mathrm{mg} / \mathrm{dL}, \mathrm{OGTT} \text { is } \\
\text { strongly recommended but is not necessary to define presence of the } \\
\text { syndrome. }\end{array}$ \\
\hline
\end{tabular}

Table 1: IDF's definition of the metabolic syndrome.

\begin{tabular}{|l|l|l|l|}
\hline Demography & Total(N=32) & Li(N=19)59.4\% & VPA (13) 40.6\% \\
\hline Gender & & & 5 \\
\hline Male (\%) & 13 & 11 & 8 \\
\hline Female (\%) & 19 & - & - \\
\hline Mean Age (years) & 36.33 & - & - \\
\hline Mean Age of Onset (years) & 23.89 & - & - \\
\hline Mean duration of Illness (years) & 12.44 & 8 & 2 \\
\hline BPAD Type I (\%) & 28 & \\
\hline
\end{tabular}

Table 2: Demography of patients with bipolar affective disorder.

\begin{tabular}{|c|c|c|}
\hline & Lithium & Valproic Acid \\
\hline Doses(mg/day) & 900 & 1015 \\
\hline Duration (Months) & 23.4 & 19 \\
\hline Serum Level (mEq/l) & 0.65 & - \\
\hline Anti-hypertensive drugs & 3 & - \\
\hline Mean high Triglycerides mmol/L & 2.1 & 0.7 \\
\hline Mean low HDL mmol/L & 0.92 & 2.5 \\
\hline Mean hyperglycaemia mmol/L Fasting & 0 & 0.047 \\
\hline Mean wide waist circumference (Inch) & 39.2 & 0.049 \\
\hline Mean weight gain (kg) & 9.8 & - \\
\hline
\end{tabular}

Table 3: Clinical parameters.

of cigarettes is smoke per day (18/day) for both Li and VPA group

\section{Discussion}

The higher prevalence of metabolic disturbances in VPA patients found in this study is consistent with the findings of previous studies involving other bipolar disorder patients [7]. Previous literature reported a 30 to $50 \%$ prevalence of metabolic syndrome in BD patients [11], which is higher than the general population seen in western countries [7]. Other studies indicated that 8 to $17 \%$ of bipolar patients have hyperglycemia and type 2 diabetes. About 17 to $36 \%$ have high triglycerides, 20 to $23 \%$ have low HDL-C, 36 to $49 \%$ have large waist circumferences, and 29 to 39\% have hypertension [7]. Although a direct causal relationship between medications and metabolic disturbance could not be determined in the current cross-sectional study, our results demonstrated that the prevalence of metabolic abnormalities in medication treated bipolar patients were similar to that in other disease groups treated with VPA and second generation antipsychotics [11]. Our study found statistically significant increase in weight by 6 months after the initiation of Li or VPA. This is consistent in a study of patients treated with lithium or VPA in combination with antipsychotic medication or placebo where weight gain was more common in the combination therapy than in monotherapy $20 \%$ vs. $6 \%$ [3].

The association of lithium and metabolic syndrome is poorly understood. However, some researchers have linked lithium and metabolic dysregulation through the effect of lithium on the hypothalamus [12]. Lithium may cause increase thirst and possible increase consumption of high caloric drinks and thus stimulating the appetite center on the hypothalamus. Lithium was also hypothesized to cause increase absorption of glucose in the adipocytes via its insulin-like effect on carbohydrate [13]. However, several studies were conducted on VPA and weight in patients with epilepsy. Therefore, a number of molecular studies were reported on VPA and metabolic syndrome. VPA is suggested to be an inhibitor of histone deacetylases (HDAC) enzyme [14]. This enzyme plays an important role in the development of metabolic syndrome through its effects on regulation of glucose metabolism and insulin homeostasis. A class of HDAC called Silent Information Regulator Types (SIRT) had been hypothesized as having a regulatory effect on stress and calorie restriction [15]. Through this mechanism, VPA is thought to suppress adiponectin levels. Hypoadiponectinaemia is associated with dyslipidemia and weight gain [16].

Useful clinical indicator for monitoring the possible metabolic abnormalities in bipolar affective patients merits further investigation. The main limitations of the present study were the relative restricted sample size and the non-prospective study design. Further prospective studies may provide solid evidence for the concerns raised in the current study.

\section{Conclusions and Recommendations}

Metabolic syndrome has become a public health problem among 
general population. Similarly, a growing concern is being expressed about this problem among mentally ill as well. Chronic mentally ill are more vulnerable for physical health problems and they show significant increase in relation to their physical health as compared to the general population [17].

Some of the changes in glucose regulation appear to be independent of weight, implicating mechanisms other than weight gain as contributing to this phenomenon. Aside from significantly decreasing medication compliance (which can prompt the exacerbation of psychotic symptoms), weight and metabolic disturbances are associated with a host of medical complications, some quite severe. Management of these adverse events involves careful consideration as to agent selection, particularly for individuals who may be independently at risk for diabetes, and close monitoring of weight, triglycerides, cholesterol, and glucose parameters during treatment. Selecting an agent with fewer propensities for weight and metabolic alteration or switching to an agent once complications have emerged is also recommended.

Participation in a diet and exercise program, possibly with a behavior modification component in order to change poor eating and lifestyle patterns, is strongly recommended. Avoiding and minimizing these adverse events will require prevention and monitoring plan that calls for active participation and collaboration between clinician and patient. With the increased emergence of diabetes and metabolic syndrome, clinicians should consider screening for hyperlipidemia or hyperglycemia annually and prior to starting or changing an antipsychotic treatment. Physicians should also consider treatments for lipid dysfunction and weight gain if they emerge during antipsychotic treatment. Although a dose-response relationship for weight gain has not been established, the development of hyperglycemia after initiation of or increase in psychotropic drugs should signal a potential metabolic syndrome. Likewise, physicians who have been worried about metabolic issues with a patient on antipsychotic or mood stabilizers should increase monitoring when raising the dose. Use of a biomarker will enable clinicians to identify metabolic changes more effectively than anthropometry and biochemistry. Adiponectin, an adipocytederived hormone, serves as a central regulatory protein in many of the physiologic pathways controlling lipid and carbohydrate metabolism. Hypoadiponectinemia is a potential biomarker of the metabolic syndrome in patients taking clozapine for schizophrenia [18]. This biomarker should be studied in patients taking mood stabilizers. Dietician has important a pivotal role in helping to improve the health of many patients by supervising balanced weight reducing diets.

$\mathrm{Li}$ and VPA are commonly prescribed medications in the maintenance phase of bipolar affective disorder. This study presented a higher prevalence of metabolic syndrome in patients taking VPA as compared to those on Li. It is important for clinicians to consider screening all patients with bipolar disorders for diabetes particularly those with risk factors for developing diabetes and those on higher risk mood stabilizers especially VPA. Collaborative engagement should include education of those identified to be at risk of metabolic syndrome. A local standardized protocol should be developed to include a periodic metabolic screening at baseline, at three months, and then annual fasting glucose. The frequency of monitoring may be increased if there are changes in fasting glucose or if risk factors change. In patients at high risk of developing diabetes consider monthly fasting blood glucose for the first three months and then check blood glucose three monthly for the first year followed by annually thereafter.

Physicians treating patients with psychiatric disorders need to be attuned to physical changes that may be a sign of serious medical conditions such as diabetes or metabolic syndrome. Physical changes such as weight gain may be an indication of metabolic side effects in patients treated with psychotropic drugs.

Patients with bipolar disorder with overweight or obesity benefit from psychological interventions, particularly behavioral and cognitivebehavioral strategies, to enhance weight reduction [19]. Furthermore, several drugs have been tested as adjunct to help reduce or, at least, control weight gain in patients with bipolar disorder. Interventions specifically designed at providing psychoeducation to patients with bipolar disorder [20] should probably be complemented with modules specifically aimed at enhancing healthy habits, reducing physical and psychiatric comorbidities. Controlled trials with larger samples are needed to understand in a better way the impact of combining educational interventions and pharmacotherapy on preventing or limiting weight gain in patients with bipolar disorder.

Key points:

- The risk of metabolic syndrome with sodium valproate is as high as atypical antipsychotics.

- Lithium has low risk of metabolic syndrome.

- Consider extra metabolic monitoring if combining sodium valproate with atypical antipsychotics.

- $\quad$ Further studies should attempt to explore pharmacogenetics of metabolic syndrome and mood stabilizers.

\section{Acknowledgement}

This work was performed as a partial fulfillment towards the International Master in Affective Neuroscience of Maastricht University and the University of Florence Supervised by Professor Joseph Zohar University of Tel Aviv, Israel.

\section{Conflict of Interest}

None.

\section{References}

1. Wirshing DA, Buckley $P$ (2003) Schizophrenia treatment challenges. Psychiatric Times 20: 1-2

2. Osby U, Correia N, Brandt L, Ekbom A, Sparen P (2000) Mortality and causes of death in schizophrenia in Stockholm county, Sweden. Schizophr Res 45 : 21-28.

3. Brown EB, McElroy SL, Keck PE, Deldar A, Adams DH, et al. (2006) A 7-week randomized, double-blind trial of olanzapinefluoxetine combination versus lamotrigine in the treatment of bipolar I depression. J Clin Psychiatry 67: 10251033.

4. Bobes J, Arango C, Arand P, Carmena R, Garcia-Garcia M, et al (2007) Cardiovascular and metabolic risk in outpatients with schizophrenia treated with antipsychotics: results of the CLAMORS study. Schizophr 90: 162-173.

5. Case CC, Jones PH, Nelson K, O'Brian Smith E, Ballantyne CM (2002) Impact of weight loss on the metabolic syndrome. Diabetes Obes Metab 4: 407-414.

6. Sanisoglu SY, Oktenli C, Hasimi A, Yokusoglu M, Ugurlu M (2006) Prevalence of metabolic Syndrome-related disorders in a large adult population in Turkey. BMC Public Health 6: 92

7. Fagiolini A, Frank E, Scott JA, Turkin S, Kupfer DJ (2005) Metabolic syndrome in bipolar disorder: findings from the Bipolar Disorder Center for Pennsylvanians. Bipolar Disord 7: 415-430.

8. Haupt DW, Newcomer JW (2002) Abnormalities in glucose regulation associated with mental illness and treatment. J Psychosom Res 53: 925-933.

9. Alberti KG, Zimmet PZ (1998) Definition, diagnosis and classification of diabetes mellitus and its complications. Part 1: Diagnosis and classification of diabetes mellitus provisional report of a WHO consultation. Diabet Med 15 539-553.

10. Silvestre JS, Prous J (2005) Research on adverse drug events, I: Muscarinic 
Citation: Abba-Aji A (2017) Prevalence of Metabolic Syndrome amongst Bipolar Patients on Lithium and Sodium Valproate Monotherapy. J Psychiatry 20: 415. doi:10.4172/2378-5756.1000415

Page 5 of 5

M3 receptor binding affinity could predict the risk of antipsychotics to induce type 2 diabetes. Methods Find Exp Clin Pharmacol 27: 289-304.

11. Elmslie JL, Porter RJ, Joyce PR, Hunt PJ, Shand BI, et al. (2009) Comparison of insulin resistance, metabolic syndrome and adiponectin in overweight bipolar patients taking sodium valproate and controls. Aust N Z J Psychiatry 43: 53-60.

12. Keck PE, McElroy SL (2003) Bipolar disorder, obesity and pharmacotherapyassociated weight gain. J Clin Psychiatry 64: 1426-1435.

13. Chen X, McMahon EG, Gulve EA (1998) Stimulatory effect of lithium on glucose transport in rat adipocytes is not mediated by elevation of IP1. Am J Physio 275: E272-E277.

14. Qiao L, Schaack J, Shao J (2006) Suppression of adiponectin gene expression by histone deacetylase inhibitor valproic acid. Endocrinology 147: 865-874.

15. McGarry JD (2002) Dysregulation of fatty acid metabolism in the etiology of type 2 diabetes. Diabetes 51: 7-18.
16. Rauchenzauner M, Laimer M, Luef G, Kaser S, Engl J, et al. (2008) Adiponectin receptor R1 is upregulated by valproic acid but not by topiramate in human hepatoma cell line, HepG2. Seizure 17: 723-726.

17. Allison DB, Mackell JA, McDonnell DD (2003) The impact of weight gain on quality of life among persons with schizophrenia. Psychiatr Serv 54: 565-567.

18. Bai YM, Chen JY, Yang WS, Chi YC, Liou YJ, et al. (2007) Adiponectin as a potential biomarker for the metabolic syndrome in Chinese patients taking clozapine for schizophrenia. J Clin Psychiatry 68: 1834-1839.

19. O'Keefe CD, Noordsy DL, Liss TB, Weiss H (2003) Reversal of antipsychoticassociated weight gain. J Clin Psychiatry 64: 907-912.

20. Colom F, Vieta E, Martinez-Aran A, Reinares M, Goikolea JM, et al. (2003) A randomized trial on the efficacy of group psychoeducation in the prophylaxis of recurrences in bipolar patients whose disease is in remission. Arch Gen Psychiatry 60: 402-407. 